\title{
Formation History of Polycyclic Aromatic Hydrocarbons in Galaxies
}

\author{
Ji Yeon Seok* \\ ASIAA \\ E-mail: jyseok@asiaa.sinica.edu.tw
}

\section{Hiroyuki Hirashita}

ASIAA

E-mail: hirashita@asiaa.sinica.edu.tw

\section{Ryosuke S. Asano}

Nagoya University

E-mail: asano.ryosukedg.mbox.nagoya-u.ac.jp

Polycyclic aromatic hydrocarbons (PAHs) are one of the major dust components in the interstellar medium (ISM). We present our model calculations for the PAH abundance in the ISM on a galaxy-evolution timescale. We consider shattering of carbonaceous dust grains as the formation mechanism of PAHs while the PAH abundance is reduced by coagulation onto dust grains, destruction by supernova shocks, and injection into star formation. We implement these processes in an one-zone chemical evolution model to obtain the evolution of the PAH abundance in a galaxy. We find that PAH formation becomes accelerated at a certain metallicity at which shattering becomes efficient. For PAH destruction, while supernova shock is a primary mechanism in the metal-poor environment, coagulation is dominant in the metal-rich environment. We compare the calculated PAH abundances with the observed abundances in galaxies with a wide metallicity range. Our models reproduce both the low PAH abundance in low metallicity galaxies and the metallicity-dependence of the PAH abundance in high-metallicity galaxies. We conclude that the observational trend can be explained by shattering of carbonaceous grains being the source of PAHs in the ISM.

The Life Cycle of Dust in the Universe: Observations, Theory, and Laboratory Experiments 18-22 November, 2013

Taipei, Taiwan

\footnotetext{
* Speaker.
} 


\section{Introduction}

A number of infrared (IR) observations have shown abundant aromatic features in spectra of various objects, and the features are commonly attributed to polycyclic aromatic hydrocarbons (PAHs). Based on various observations, it is found that there is a trend that the luminosity of PAH emission depends on metallicity. As the metallicity of galaxies decreases, the measured luminosity of PAH emission decreases [1]. Similarly, PAH abundances of a large galaxy sample are derived showing that PAH abundances of low metallicity galaxies are lower than those of high metallicity galaxies [2]. In particular, it is noticeable that some low-metallicity galaxies with active starformation such as blue compact dwarf galaxies (BCDs; e.g., [3]) show a dearth of PAH features because starburst galaxies generally show rich PAH emission.

To interpret this observational trend, several hypotheses have been proposed. There can be two different approaches; one is that PAHs in low-metallicity galaxies are intrinsically deficient probably due to the extreme youth of galaxies. [4] explained the lack of PAHs in low metallicity galaxies with the delayed injection of carbon dust from asymptotic giant branch (AGB) stars. Another explanation is that PAH carriers are more efficiently destroyed in a low-metallicity environment. Since star formation in low-metallicity galaxies is dominated by more massive stars compared to that in high-metallicity galaxies, the PAH destruction by supernova ( $\mathrm{SN}$ ) shocks is more severe. Since ionic line ratios such as [Fe II]/[Ne II] trace SN shocks, then if these shocks are responsible for destruction, then one would expect an anti-correlation between these ratios and PAH intensity; indeed such an anti-correlation is seen in a sample of starburst galaxies with a wide range of metallicity [5]. Although there is no clear explanation for the observational trend between the metallicity and PAH abundance, it is likely that the metallicity dependence of PAH abundance originates from PAH formation and/or destruction mechanisms. Hence, it is important to know what controls PAH abundance and how it is correlated with metallicity.

In general, chemical reactions in the atmospheres of carbon-rich stars are considered to be a major laboratory to form PAHs. In addition to this, another mechanism is proposed, which is the formation of PAHs (or PAH-like very small carbonaceous grains) by fragmentation of larger grains by shattering in shocks [6]. The size distribution of grains is calculated for a dense region with a density of $10^{5} \mathrm{~cm}^{-3}$ where C-type shocks with a velocity of $30 \mathrm{~km} \mathrm{~s}^{-1}$ are interacting [7]. This work showed that lots of small grains (overlapping with the PAH-size range) are produced by shattering. Furthermore, grain shattering can be a more general process. Grain shattering in the turbulent interstellar medium (ISM) is examined [8] which found that significant small grains can form by shattering, particularly, in the warm ionized medium (WIM).

From an observational point of view, there is not much direct evidence of PAH formation by fragmentation. There have been only few detections of PAH emission in supernova remnants (SNRs). For example, using the AKARI satellite, we performed coarse spectral mapping of the SNR N49 in the Large Magellanic Cloud (LMC), which is interacting with a molecular cloud [9]. We confirmed the $3.3 \mu \mathrm{m}$ PAH emission is related to the SNR although the detection of the PAH emission in $\mathrm{N} 49$ does not guarantee the formation of PAHs in shocked regions. Recently, however, a laboratory experiment found that spectroscopic properties of heated hydrogenated amorphous carbons (HACs) are very similar to those of PAHs [10]. The laboratory spectra nicely reproduce both the $3.3 \mu \mathrm{m}$ aromatic feature and the weak $3.4 \mu \mathrm{m}$ aliphatic feature that we observed. They 
suggested the aromatization due to the destructive thermal processing of HACs may be an early stage in the formation of PAH clusters. Therefore, it is worth revisiting the formation of PAHs through fragments under a new motivation of turbulence driven shattering and to see how metallicity is correlated with PAH processing in a galactic timescale.

\section{PAH evolution model}

In our model, we focus on the shattering of carbonaceous dust grains due to the interstellar turbulence as the PAH formation mechanism. For destruction, astration, shock destruction, and coagulation of grains are included. We determine the timescales of shattering, coagulation, and SN destruction, and calculate the time evolution of the total PAH mass in a galaxy. Note that the evolution of the total PAH mass depends on the evolution of the total dust mass because dust grains are used in shattering and coagulation. We adopt the chemical evolution model of [11], following the build up of metals and dust over time. Stellar dust production as well as dust mass growth in the ISM are considered as dust formation processes while SN shocks and astration are included as destruction mechanisms. In the model of [11], grain growth is inefficient at low metallicities and becomes efficient at a certain metallicity referred to as "critical metallicity".

To derive shattering and coagulation timescales, we use two initial size distributions of grains. One is a simple power-law distribution with enhancement at the size range of PAHs. This is based on the grain size distribution of [14] (hereafter MRN), which is derived from the observed interstellar extinction curve. We simply extrapolate the MRN size distribution down to the PAH-size regime and normalize it to be consistent with the Galactic PAH abundance. We refer this size distribution to "MRN+enhanced PAH", which is in fact similar to the more elaborate calculation by [12]. Another size distribution is taken from [13] (hereafter WD01), which has two log-normal size distributions for PAHs and very small grains. Using the two size distributions, we examine their evolution by shattering and coagulation in each ISM phase adopting the formulation in [8]. (Shattering occurs in WIM most efficiently whereas coagulation is more efficient in dense clouds (DC).) We fit the variation of the total PAH mass density with time and derive the timescale. Then, we obtain the timescales of the entire ISM by weighted summation for the mass fraction of each ISM phase. More details of the calculations are explained in [15].

\section{Evolution of PAH abundance in galaxies}

We present the variation of the PAH and dust abundances $\left(Z_{\mathrm{PAH}}\right.$ and $Z_{\text {dust }}$ with solid and dashed lines, respectively) as a function of the gas metallicity $\left(Z_{\mathrm{gas}}\right)$ for the star formation timescale of $\tau_{\mathrm{SF}}=5 \mathrm{Gyr}$ in Figure 1 (top). The total mass ratio of PAH to dust is also shown in Figure 1 (bottom). For the WD01 size distribution, the PAH abundance and the mass ratio are overlaid with dotted lines. The general trend from our model is that at low metallicity, the PAH abundance is very low and slowly increases until $Z_{\text {gas }} \approx 0.1 Z_{\odot}$. Later, the PAH abundance is rapidly enhanced as the dust abundance rapidly increases, which is mainly caused by grain growth associated with the critical metallicity [11]. Then, the enhancement slows down at $Z_{\text {gas }} \approx 0.3 Z_{\odot}$ when grain growth is saturated due to complete accretion of gas-phase metals onto dust. 


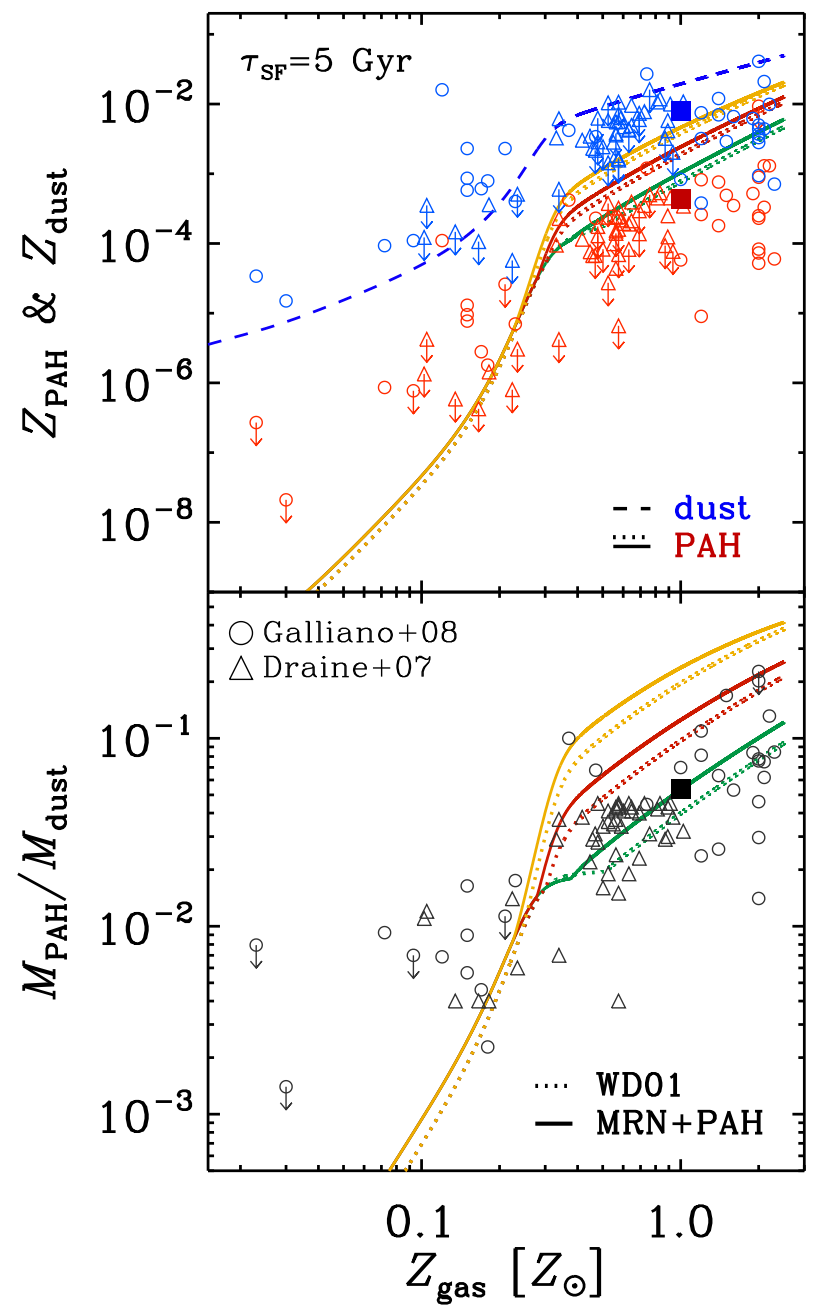

Figure 1: Top: Calculated PAH-to-gas mass ratio ( $Z_{\mathrm{PAH}}$, solid line) and dust-to-gas ratio $\left(Z_{\text {dust }}\right.$, dashed line) as a function of metallicity $\left(Z_{\mathrm{gas}}\right)$ for $\tau_{\mathrm{SF}}=5 \mathrm{Gyr}$. For $Z_{\mathrm{PAH}}$, the cases for $\tau_{\mathrm{DC}}=1,3$, and $10 \mathrm{Myr}$ are overlaid in each panel with green, red, and yellow lines, respectively. Those calculated for the WD01 size distribution are overlaid with dotted lines. For comparison, observational data from [2] and [4] are shown with triangles and circles, respectively. $Z_{\mathrm{PAH}}$ and $Z_{\mathrm{dust}}$ are distinguished with red and blue symbols. Also, $Z_{\mathrm{PAH}}$ and $Z_{\mathrm{dust}}$ of the diffuse Galactic ISM [12] are denoted with red and blue squares, respectively. Bottom: Total mass ratio of PAH to dust $\left(M_{\mathrm{PAH}} / M_{\mathrm{dust}}=Z_{\mathrm{PAH}} / Z_{\mathrm{dust}}\right)$ for $\tau_{\mathrm{SF}}=5 \mathrm{Gyr}$.

We examine the effect of the lifetime of a dense cloud $\left(\tau_{\mathrm{DC}}\right)$. In Figure 1, three cases of $\tau_{\mathrm{DC}}=$ 1,3 , or $10 \mathrm{Myr}$, are shown with green, red, and yellow lines, respectively. The different $\tau_{\mathrm{DC}}$ affect the evolution of the PAH abundance at the late stage $\left(Z_{\text {gas }} \gtrsim 0.3 Z_{\odot}\right)$ when coagulation becomes efficient. As the dust abundance increases, the coagulation timescale $\left(\tau_{\text {coag }}\right)$ rapidly decreases. Since $\tau_{\text {coag }}$ of a dense cloud cannot exceed the lifetime of the cloud $\left(\tau_{\mathrm{DC}}\right)$, coagulation is restricted by $\tau_{\mathrm{DC}}$. In addition, the different size distributions (MRN+enhanced PAH and WD01) do not result in significant differences. With WD01, the PAH abundance is less than that of MRN+enhanced PAH because of the slightly shorter coagulation timescale. 
The model calculations are compared to observational data of galaxies with diverse metallicities $[2,4]$ in Figure 1. Our results are consistent with the observed PAH and dust abundances, which reproduce both the paucity of PAHs in low metallicity and the rapid increase in PAH abundance above a certain metallicity. This is a natural consequence that PAH formation and destruction mechanisms in our models depend on the dust abundance. Both shattering and coagulation are not efficient in low-metallicity galaxies because the dust abundance is insufficient. At low metallicities, dust production is dominated by stellar sources such as SNe or AGB stars, of which dust yield does not strongly depend on metallicity [11]. On the other hand, dust growth by accretion in the ISM becomes efficient after the metallicity of a galaxy exceeds the critical metallicity, then it dominates the dust production in the galaxy afterward. This strong metallicity dependence of the dust production via grain growth is reflected in the strong metallicity dependence of the PAH abundance. The advantage of our models is that the strong metallicity dependence of the PAH abundance is naturally explained.

\section{Summary}

We test our PAH evolution models taking into account PAH formation by shattering in turbulence. We consider destruction in shocks, coagulation, and astration as PAH destruction mechanisms. In our calculations, PAH evolution via shattering and coagulation in a galaxy explains both the paucity of PAHs at low-metallicities and the rapid enrichment of PAHs at high-metallicities. This is a natural consequence of PAH formation and destruction mechanisms that depend on dust abundance. When the metallicity of a galaxy is greater than the critical metallicity, grain growth in the ISM is a dominant origin of dust, and the rate of grain growth has strong dependence on metallicity [11]. We explain the metallicity-dependence of the PAH abundance in galaxies with our models, which supports the validity of shattering in turbulence as a PAH formation mechanism in the ISM.

\section{References}

[1] S. C. Madden, F. Galliano, A. P. Jones, \& M. Sauvage, ISM properties in low-metallicity environments, A\&A 2006 (446) 877

[2] B. T. Draine, D. A. Dale, G. Bendo, et al., Dust masses, PAH abundances, and starlight intensities in the SINGS galaxy sample, ApJ 2007 (663) 866

[3] L. K. Hunt, T. X. Thuan, Y. I. Izotov, \& M. Sauvage, The Spitzer view of low-metallicity star formation. III. fine-structure lines, aromatic Features, and molecules, ApJ 2010 (712) 164

[4] F. Galliano, E. Dwek, \& P. Chanial, Stellar evolutionary effects on the abundances of polycyclic aromatic hydrocarbons and supernova condensed dust in galaxies, ApJ 2008 (672) 214

[5] B. O'Halloran, S. Satyapal, \& R. P. Dudik, The polycyclic aromatic hydrocarbon emission deficit in low-metallicity galaxies-A Spitzer view, ApJ 2006 (641) 795

[6] A. P. Jones, A. G. G. M. Tielens, \& D. J. Hollenbach, Grain shattering in shocks: The interstellar grain size distribution, ApJ 1996 (469) 740

[7] V. Guillet, G. Pineau des Forêts, \& A. P. Jones, Shocks in dense clouds III. Dust processing and feedback effects in C-type shocks, A\&A 2011 (527) A123 
[8] H. Hirashita, \& H. Yan, Shattering and coagulation of dust grains in interstellar turbulence, MNRAS 2009 (394) 1061

[9] J. Y. Seok, B.-C. Koo, \& T. Onaka, Detection of the $3.3 \mu$ m aromatic feature in the supernova remnant N49 with AKARI, ApJ 2012 (744) 160

[10] K. A. K. Gadallah, H. Mutschke, \& C. Jäger, Analogs of solid nanoparticles as precursors of aromatic hydrocarbons, A\&A 2013 (554) A12

[11] R. S. Asano, T. T. Takeuchi, H. Hirashita, \& A. K. Inoue, Dust formation history of galaxies: A critical role of metallicity for the dust mass growth by accreting materials in the interstellar medium, Earth, Planets, and Space 2013 (65) 213

[12] V. Zubko, E. Dwek, \& R. G. Arendt, Interstellar dust models consistent with extinction, emission, and abundance constraints, ApJS 2004 (152) 211

[13] J. C. Weingartner, \& B. T. Draine, Dust grainĐsize distributions and extinction in the Milky Way, Large Magellanic Cloud, and Small Magellanic Cloud, ApJ 2001 (548) 296

[14] J. S. Mathis, W. Rumpl, \& K. H. Nordsieck, The size distribution of interstellar grains, ApJ 1977 (217) 425

[15] J. Y. Seok, H. Hirashita, \& R. S. Asano, Formation history of polycyclic aromatic hydrocarbons in galaxies, accepted to MNRAS 2014 